Review

\title{
A systematic review and meta-analysis on the effect of neoadjuvant chemotherapy on complications following immediate breast reconstruction
}

\author{
Jajini Varghese ${ }^{\mathrm{a}, 1}$, Shireen S. Gohari ${ }^{\mathrm{b}, \mathrm{c}, 1}$, Hirah Rizki ${ }^{\mathrm{f}}$, Ichael Faheem ${ }^{\mathrm{c}}$, \\ Benjamin Langridge ${ }^{\mathrm{a},{ }^{*}}$, Sherko Kümmel ${ }^{\mathrm{d}}$, Laura Johnson ${ }^{\mathrm{c}}$, Peter Schmid ${ }^{\mathrm{e}}$ \\ ${ }^{a}$ Royal Free Hospital and Division of Surgery and Interventional Science, UCL, United Kingdom \\ ${ }^{\mathrm{b}}$ Barts and the London School of Medicine, London, United Kingdom \\ ' St Bartholomew's Hospital, London, United Kingdom \\ ${ }^{\mathrm{d}}$ Kliniken Essen-Mitte, E6ssen, Germany \\ e Barts Cancer Institute, Centre for Experimental Cancer Medicine, Queen Mary University of London, London, United Kingdom \\ ${ }^{\mathrm{f}}$ Royal Marsden Hospital, United Kingdom
}

\section{A R T I C L E I N F O}

\section{Article history:}

Received 30 October 2020

Accepted 30 November 2020

Available online 3 December 2020

\section{Keywords:}

Immediate breast reconstruction

Neoadjuvant chemotherapy

Post-operative complications

Oncoplastic surgery

Plastic and reconstructive surgery

Breast surger

\begin{abstract}
A B S T R A C T
Background: The impact of neoadjuvant chemotherapy (NACT) on surgical outcomes following immediate breast reconstruction (IBR) remains unclear. While it is generally considered safe practice to perform an IBR post NACT, reported complication rates in published data are highly variable with the majority of studies including fewer than 50 patients in the NACT and IBR arm. To evaluate this further, we conducted a systematic review and meta-analysis on the effect of NACT on autologous and implant based immediate breast reconstructions. We aimed to assess for differences in the post-operative course following IBR between patients who received NACT with those who did not.

Methods: PubMed, EMBASE, and Cochrane Library were searched from 1995 to Sept 2, 2020 to identify articles that assessed the impact of NACT on IBR. All included studies assessed outcomes following IBR. Only studies comparing reconstructed patients receiving NACT to a control group of women who did not receive NACT were included. Unadjusted relative risk of outcomes between patients who received or did not receive NACT were synthesized using a fixed-effect meta-analysis. The evidence was assessed using the Newcastle Ottawa Scale scores and GRADE. Primary effect measures were risk ratios (RRs) with 95\% confidence intervals.

Results: A total 17 studies comprising 3249 patients were included in the meta-analyses. Overall, NACT did not increase the risk of complications after immediate breast reconstructions (risk ratio [RR]: 0.91, $95 \% \mathrm{CI} 0.74$ to $1.11, p=0.34$ ). There was a moderate, but not significant, increase in flap loss following NACT compared with controls (RR: $1.23,95 \%$ CI 0.70 to $2.18, p=0.47 ; I^{2}=0 \%$ ). Most notably, there was a statistically significant increase in implant/expander loss after NACT (RR: $1.54,95 \%$ CI 1.04 to 2.29, $p=0.03 ; I^{2}=34 \%$ ). NACT was not shown to significantly increase the incidence of hematomas, seromas or wound complications, or result in a significant delay to commencing adjuvant therapy (RR: $1.59,95 \%$ CI 0.66 to $3.87, p=0.30$ ).

Conclusion: Immediate breast reconstruction after NACT is a safe procedure with an acceptable postoperative complication profile. It may result in a slight increase in implant loss rates, but it does not delay commencing adjuvant therapy.
\end{abstract}

() 2020 The Authors. Published by Elsevier Ltd. This is an open access article under the CC BY-NC-ND license (http://creativecommons.org/licenses/by-nc-nd/4.0/).

Contents

\footnotetext{
* Corresponding author.

E-mail address: benjamin.langridge.14@ucl.ac.uk (B. Langridge).

1 joint co-authors.
} 


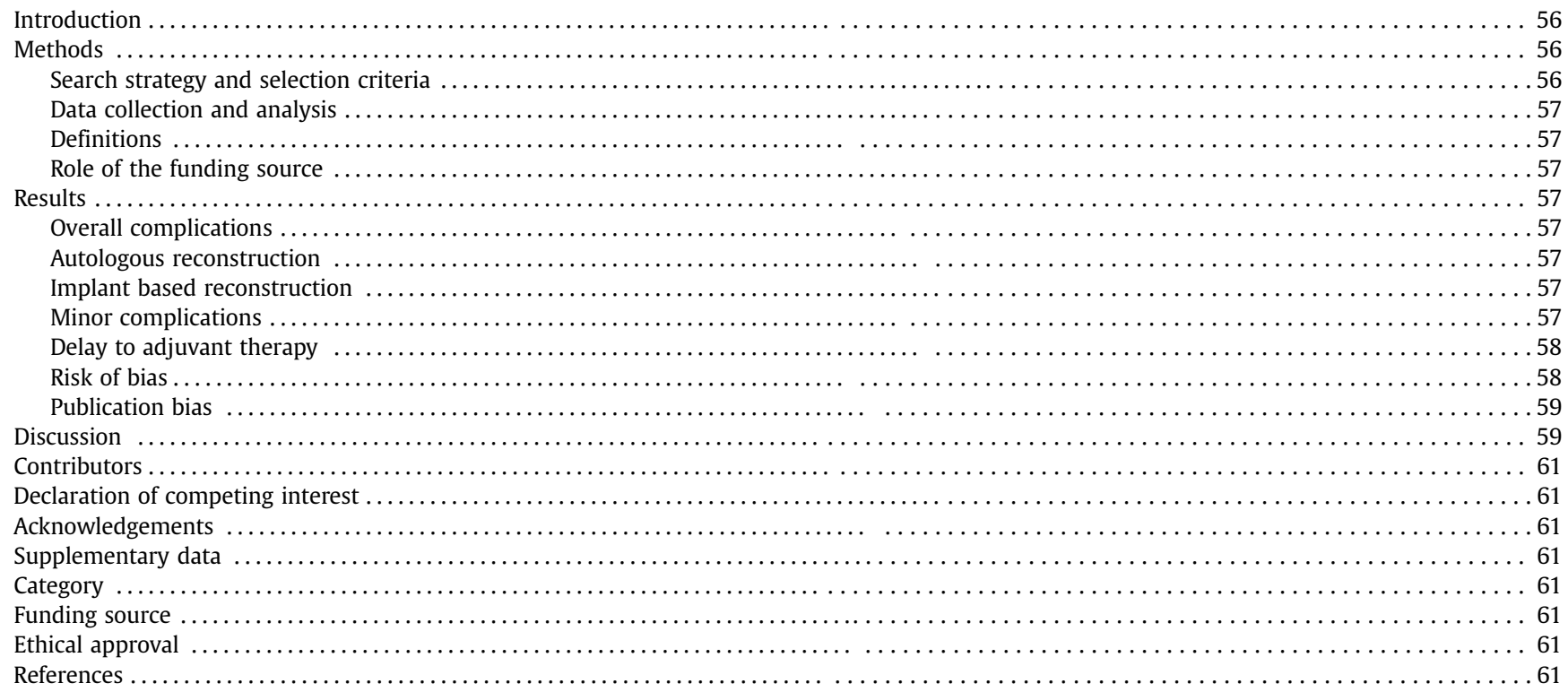

\section{Introduction}

Despite efforts at early detection, locally advanced breast cancer remains a challenge for achieving local and distant control of disease [1,2]. Over the last decade, the use of neoadjuvant chemotherapy (NACT) has been increasing. This approach allows early evaluation of response to therapy, guides the delivery of adjuvant treatment such as TDM1, and most significantly, can de-escalate surgery from a mastectomy to breast conserving surgery [1,3] [-] [5]. However, approximately two-thirds of women will still require a mastectomy on completion of NACT [6]. Accompanying a mastectomy with an immediate breast reconstruction (IBR) has welldocumented benefits on aesthetic results, quality of life and psychological outcomes. While reports on the impact of NACT on surgical outcomes are highly variable, it is generally considered safe practice to perform an IBR following NACT. Current UK based practice is to offer reconstruction to all women who are oncologically and medically stable, but data to support this practice in the post-NACT setting is limited [7,8].

Post-mastectomy breast reconstructions have become increasingly common practice, with a trend towards immediate implant based reconstructions [9]. While this reconstructive modality provides satisfactory aesthetic results, autologous tissue reconstructions can offer a more natural shape and feel with higher reported rates of patient satisfaction [10]. Patient and oncological factors are taken into consideration prior to selecting the most suitable reconstruction for each patient. For example, estimated mastectomy volume, degree of ptosis, volume available for an autologous reconstruction and degree of skin involvement from the cancer. Theoretically, the post-operative course following either of these reconstructive methods can be influenced by the effect of cytotoxic chemotherapy agents on small vessel endothelium and the patient's immune system, resulting in loss of the reconstruction [11].

Delayed and problematic wound healing has been seen in animal studies secondary to chemotherapy drugs $[12,13]$. There findings have not been evidenced in clinical practice, as reconstructions are typically scheduled several weeks after chemotherapy is completed, allowing the recovery of a patient's immune system prior to surgery [14]. Despite this, a recent study reported that women who receive NACT are less likely to be offered an immediate breast reconstruction [15].

The objective of this systematic review and meta-analysis is to assess the impact of NACT on patients undergoing autologous or implant reconstruction following NACT. The impact of NACT on delays to adjuvant therapy was also evaluated. The aim was to synthesize available evidence to support the safety of immediate breast reconstruction following NACT by comparing the postoperative course of patients who underwent an IBR following NACT with those who underwent an IBR without NACT.

\section{Methods}

\section{Search strategy and selection criteria}

A systematic review and meta-analysis was conducted according to the Preferred Reporting Items for Systematic Reviews and Meta-analyses (PRISMA) guidelines [16]. Studies published from 1995 to Sept 2, 2020 that examined the effects of NACT on postoperative complications following autologous or implant-based breast reconstructions were included. No language restrictions were placed on the search. This review was prospectively registered in the PROSPERO database (Registration Number CRD42020183761). PubMed, EMBASE and Cochrane Library databases were searched. Full details of the search strategy used for PubMed, EMBASE and Cochrane Library are in the supplementary material. Authors JV and SG independently screened titles and abstracts to identify articles meeting the eligibility criteria. Full-text articles were reviewed independently by JV and SG, with discrepancies discussed by three authors (JV, SG, and MF) to reach consensus. References of selected articles were screened to identify additional relevant papers.

Where not available in the publication, study authors were contacted for individual patient-level data to differentiate between complications occurring in patients receiving NACT compared to controls. Of the four corresponding authors we contacted, one provided information on partial flap loss, which we included in the meta-analysis. 
Case reports, and full text articles with insufficient information were excluded. We included studies reporting at least one major complication in female subjects of any age that had breast surgery (mastectomy and autologous flap or implant-based reconstruction).

\section{Data collection and analysis}

The data were extracted independently by three authors (JV, SG and MF) including authors, year of publication, study design, number of subjects, age (mean, median, and range), follow-up period, NACT regimen. The type of reconstruction and reported complications were recorded for all patients. Results were divided into two groups: the NACT group, who received NACT prior to their IBR, and the control group who did not receive NACT prior to their IBR. Data regarding complications were divided into major and minor complications as per the definitions below.

The timeline for the occurrence complications was not always clear in the reports. Subset analyses based on follow-up period were not possible as there was a wide range (six weeks to 52 months), with only two studies reporting on prospective data for six weeks after surgery.

Review Manager 5.3 was used to record the review process and statistical analysis. Fixed effect meta-analysis was performed using the Mantel-Haenszel approach. Evidence for departure from the fixed-effects model was assessed using Higgins and Thompson's $I^{2}$ statistic [17]. The primary effect measures were risk ratios (RRs) with 95\% confidence intervals (CIs). All statistical analyses were two-sided. Individual study risk of bias and quality was assessed using the Newcastle-Ottawa Scale (NOS). Potential publication bias was evaluated by visual assessment of funnel plots for each metaanalysis endpoint.

\section{Definitions}

Major complications were defined as any of the following: total flap loss, partial flap loss, implant/expander (I/E) loss, and mastectomy skin flap necrosis. Minor complications were defined as any of the following: hematoma, seroma, infection, fat necrosis, and wound complications. Wound complications were further defined as any of the following: dehiscence, delayed healing, epidermolysis, donor site breakdown, and infection. Flap loss was defined as loss of circulation in the autologous flap. Implant loss was defined as removal of prosthesis without immediate replacement. A hematoma was defined as a collection of blood within a breast that required surgical management. A seroma was defined as fluid collection that warranted aspiration. Infection was defined as localized or systemic evidence of infection that led to administration of antibiotics.

\section{Role of the funding source}

There was no funding source for this study. The corresponding author had full access to all data and the final responsibility for the decision to submit for publication.

\section{Results}

The database searches identified a total of 447 publications. Following exclusion of duplicates and irrelevant abstracts, 99 fulltext articles were screened. A total of 17 studies, published between 1995 and 2020, that examined the influence of NACT on immediate breast reconstruction and contained extractable data, were included in the meta-analysis (Supplementary Fig. 1).

Data on outcomes were extracted from 17 studies and included a total of 3249 patients, of which 575 patients received NACT (NACT group) and 2674 patients did not receive NACT (control group). Five studies assessed free-flap reconstructions exclusively [18] [-] [22], six studies looked at implant-based reconstructions only [23-28], and the remaining six studies reported on a combination of autologous and implant-based reconstructions [29-34].

The included studies were deemed to be of moderate quality overall with ranking $\geq 5$ stars on the modified NOS but were scored 'Very Low' on the Grading of Recommendations, Assessment, Development and Evaluations (GRADE) scoring protocol.

The main characteristics of all included studies and NOS scores are provided in Supplementary Table 1. Supplementary Table 2 displays the data on major and minor outcomes extracted from the included studies. Supplementary Table 3 provides a summary of findings for each outcome (GRADE Table).

\section{Overall complications}

Of the 17 studies in the meta-analysis, nine $(n=1351$ patients; NACT $n=270$, Controls $n=1081$ ) reported data on overall complications [19,20,23-25,27,28,30,34]. There was little evidence of difference in incidence of overall complications between women who received NACT and those who did not (RR: $0.91,95 \% \mathrm{CI}: 0.74$ to $1.11, p=0.34$; Fig. 1).

\section{Autologous reconstruction}

Five studies reported on reconstruction loss after a free-flap autologous based reconstruction $(\mathrm{n}=935$ patients; NACT $n=182$, control $n=753$ ). The pooled analysis suggests little difference between flap loss rates (total or partial) for patients receiving NACT compared with the control group, although the $\mathrm{CI}$ was relatively wide (RR: $0.94 ; 95 \%$ CI 0.46 to $1.94, p=0.87$; Fig. 2 A). One study reported only on complications following pedicle flaps with an implant reconstruction. They reported 1 flap loss in the NACT group only $(n=102$ patients; NACT $n=33$, Control $n=69$ ) [30].

\section{Implant based reconstruction}

Our pooled estimate from five studies ( $\mathrm{n}=868$ patients; NACT, $\mathrm{n}=161$, Controls $\mathrm{n}=707$ ) showed some evidence for increase in implant loss among patients receiving NACT compared with the control group although the CI was wide (RR: $1.54,95 \%$ CI 1.04 to 2.29, $p=0.03$; Fig. 2B).

However, when we limited the analyses to data from two studies that had follow-up of 6 weeks; thereby negating the effect of adjuvant therapy on this outcome, there was no significant effect [25,27]. Peled et al., followed up patients for 19 months and reported $59 \%$ of patients undergoing NACT had postoperative radiation therapy compared to $36 \%$ of patients treated with adjuvant chemotherapy $(p=0.05)$. Despite this difference, the NACT group did not have a significantly greater implant loss rate.

\section{Minor complications}

Separate meta-analyses were performed to obtain pooled estimates for risk of hematomas, seromas, and wound complications. From the 12 studies ( $\mathrm{n}=1956$ patients) that reported on the influence of NACT on the occurrence of hematoma after surgery, the pooled result showed little difference in the incidence of hematoma between the two groups ( $R R=0.99 ; 95 \% \mathrm{CI} 0.61$ to $1.60, p=0.97$; Fig. 3A).

Similarly, administration of NACT did not affect the incidence of seromas ( $\mathrm{RR}=0.86 ; 95 \% \mathrm{CI} 0.58$ to $1.28, p=0.46$; Fig. $3 \mathrm{~B})$. Analyses 


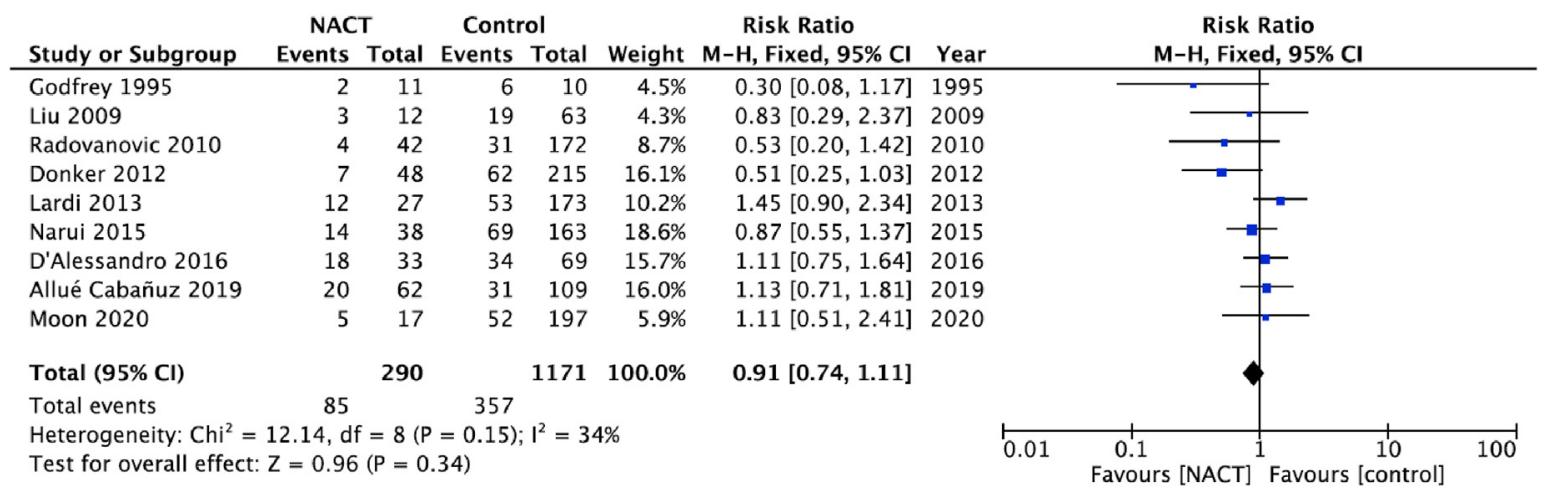

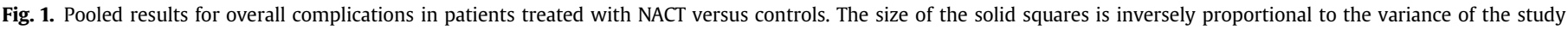
estimate. The diamond represents the fixed effects risk ratio and $95 \%$ confidence interval.

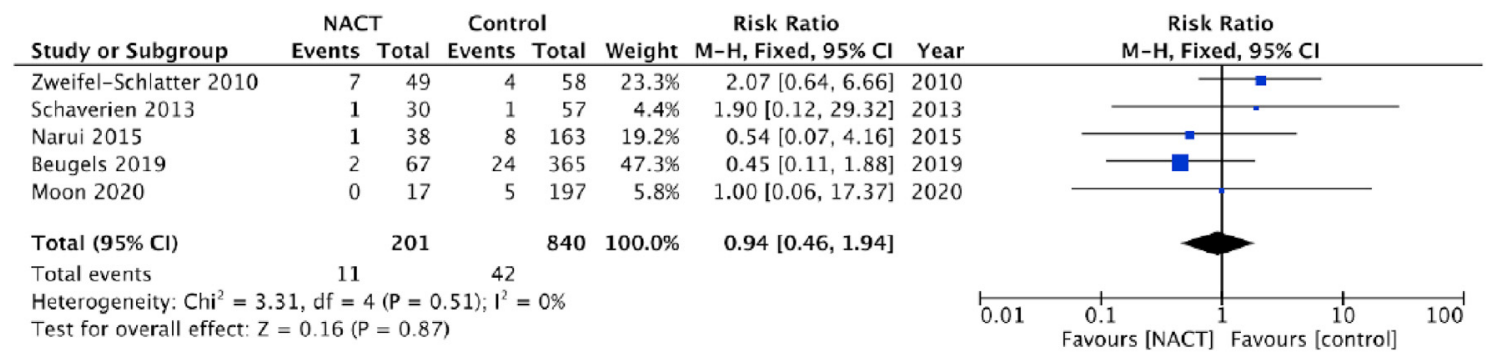

A. Flap losses (total and partial) in free-flap reconstructions

\begin{tabular}{|c|c|c|c|c|c|c|c|c|c|c|c|}
\hline \multirow[b]{2}{*}{ Study or Subgroup } & \multicolumn{2}{|c|}{ NACT } & \multicolumn{2}{|c|}{ Control } & \multicolumn{3}{|c|}{ Risk Ratio } & \multirow{2}{*}{\multicolumn{4}{|c|}{$\begin{array}{c}\text { Risk Ratio } \\
\text { M-H, Fixed, } 95 \% \mathrm{Cl}\end{array}$}} \\
\hline & Events & Total & Events & Total & Weight & M-H, Fixed, 95\% Cl & Year & & & & \\
\hline Radovanovic 2010 & 3 & 42 & 9 & 172 & $12.5 \%$ & $1.37[0.39,4.82]$ & 2010 & & & & \\
\hline Peled 2010 & 8 & 31 & 8 & 45 & $23.1 \%$ & $1.45[0.61,3.45]$ & 2010 & & & & \\
\hline Jiménez-Puente 2011 & 7 & 13 & 19 & 102 & $15.2 \%$ & $2.89[1.51,5.52]$ & 2011 & & & & \\
\hline Donker 2012 & 4 & 48 & 24 & 215 & $31.0 \%$ & $0.75[0.27,2.05]$ & 2012 & & - & & \\
\hline Lardi 2013 & 6 & 27 & 19 & 173 & $18.2 \%$ & $2.02[0.89,4.61]$ & 2013 & & & & \\
\hline Total $(95 \% \mathrm{Cl})$ & & 161 & & 707 & $100.0 \%$ & $1.54[1.04,2.29]$ & & & & & \\
\hline Total events & 28 & & 79 & & & & & & & & \\
\hline $\begin{array}{l}\text { Heterogeneity: } \mathrm{Chi}^{2}= \\
\text { Test for overall effect: }\end{array}$ & $\begin{array}{l}07, \mathrm{df}= \\
=2.17\end{array}$ & $\begin{array}{l}4(P= \\
=0 .\end{array}$ & $\begin{array}{l}0.19) ; 1^{2} \\
\text { 3) }\end{array}$ & $=34 \%$ & & & & 0.01 & $\begin{array}{c}0.1 \\
\text { Favours [NACT] }\end{array}$ & 1 Favours [control] & 100 \\
\hline
\end{tabular}

B Implant or Expander losses

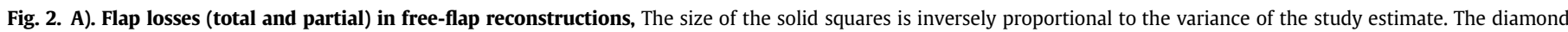

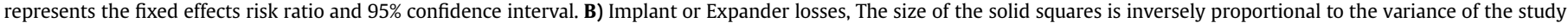
estimate. The diamond represents the fixed effects odds ratio and $95 \%$ confidence interval.

of wound complications alone (dehiscence, delayed healing, epidermolysis, donor site breakdown, and infection) for all reconstructions found the administration of NACT was not associated with significantly increased risk $(R R=1.15 ; 95 \%$ CI 0.92 to 1.44 , $p=0.22$; Fig. 3B).

\section{Delay to adjuvant therapy}

Four studies reported on delays to adjuvant therapy (Fig. 4) and the pooled estimate showed no statistically significant difference between women receiving NACT compared with those who did not (RR: 1.59, 95\% CI 0.66 to 3.87, $p=0.30$; Fig. 4) [21,25,29,32].

\section{Risk of bias}

The most common bias evident in the reports was in patient selection, whereby reconstruction after NACT was offered to significantly younger women $[18,20,21,25]$. In the study by Beugels et al., the control group had more smokers, leading to a protective association with NACT exposure. Although they reported adjusted risk estimates, these were not used in the present meta-analysis as they were not available for the other studies [18]. Disease was significantly more advanced in the NACT group compared with the control group in some studies [20-22]. The follow-up periods varied widely in the studies ( 6 weeks -52 months, Supplementary Table 1). In addition, the impact of recall bias and record accuracy could be an issue with retrospective studies in general [18-20,24,26,28-33].

We used NOS score and the GRADE table to assess quality of studies. The NOS scores ranged from moderate to high quality $(\geq 5$; Supplementary Table 1; supplementary 2-18), while the GRADE scores were severely affected by the observational study designs and patient selection bias and were scored as 'Very Low' (Supplementary Table 3). 


\begin{tabular}{|c|c|c|c|c|c|c|c|c|c|c|c|}
\hline Study or Subgroup & \multicolumn{2}{|c|}{ NACT } & $\begin{array}{c}\text { Contr } \\
\text { Events }\end{array}$ & $\begin{array}{l}\text { rol } \\
\text { Total }\end{array}$ & \multicolumn{3}{|c|}{ Risk Ratio } & \multicolumn{4}{|c|}{$\begin{array}{c}\text { Risk Ratio } \\
\mathrm{M}-\mathrm{H} \text {, Fixed, } 95 \% \mathrm{Cl}\end{array}$} \\
\hline Liu 2009 & 1 & 12 & 3 & 63 & $2.8 \%$ & $1.75[0.20,15.44]$ & 2009 & & & & \\
\hline Azzawi 2010 & 0 & 58 & 1 & 140 & $2.6 \%$ & $0.80[0.03,19.27]$ & 2010 & & & & \\
\hline Radovanovic 2010 & 0 & 42 & 2 & 172 & $2.9 \%$ & $0.80[0.04,16.45]$ & 2010 & & & & \\
\hline Peled 2010 & 5 & 57 & 1 & 65 & $2.7 \%$ & $5.70[0.69,47.38]$ & 2010 & & & & \\
\hline Zweifel-Schlatter 2010 & 3 & 49 & 2 & 58 & $5.4 \%$ & $1.78[0.31,10.20]$ & 2010 & & & & \\
\hline Donker 2012 & 0 & 48 & 11 & 215 & $12.5 \%$ & $0.19[0.01,3.20]$ & 2012 & & & & \\
\hline Schaverien 2013 & 2 & 33 & 3 & 62 & $6.1 \%$ & $1.25[0.22,7.13]$ & 2013 & & & & \\
\hline Lardi 2013 & 2 & 21 & 8 & 128 & $6.6 \%$ & $1.52[0.35,6.69]$ & 2013 & & & & \\
\hline Narui 2015 & 1 & 40 & 12 & 165 & $13.7 \%$ & $0.34[0.05,2.57]$ & 2015 & & & & \\
\hline D'Alessandro 2016 & 2 & 33 & 0 & 69 & $1.0 \%$ & $10.29[0.51,208.55]$ & 2016 & & & & \\
\hline Beugels 2019 & 3 & 67 & 34 & 365 & $30.9 \%$ & $0.48[0.15,1.52]$ & 2019 & & 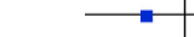 & & \\
\hline Allué Cabañuz 2019 & 4 & 62 & 6 & 109 & $12.8 \%$ & $1.17[0.34,3.99]$ & 2019 & & & & \\
\hline Total $(95 \% \mathrm{CI})$ & & 522 & & 1611 & $100.0 \%$ & $0.99[0.61,1.60]$ & & & & & \\
\hline Total events & 23 & & 83 & & & & & & & & \\
\hline $\begin{array}{l}\text { Heterogeneity: } \mathrm{Chi}^{2}=1 \\
\text { Test for overall effect: } Z\end{array}$ & $\begin{array}{l}3, \mathrm{df}= \\
0.04(P\end{array}$ & $\begin{array}{l}11(P= \\
=0.97\end{array}$ & $=0.53)$ & $1^{2}=0 \%$ & & & & 0.01 & $\begin{array}{c}0.1 \\
\text { Favours [NACT] }\end{array}$ & $\frac{10}{\text { Favours [control] }}$ & 100 \\
\hline
\end{tabular}

\section{A. Haematomas in all reconstructions}

\begin{tabular}{|c|c|c|c|c|c|c|c|c|c|c|}
\hline Study or Subgroup & \multicolumn{2}{|c|}{ NACT } & \multicolumn{2}{|c|}{ Control } & \multicolumn{3}{|c|}{ Risk Ratio } & \multicolumn{3}{|c|}{$\begin{array}{c}\text { Risk Ratio } \\
\text { M-H, Fixed, 95\% Cl }\end{array}$} \\
\hline Liu 2009 & 1 & 12 & 2 & 63 & $0.6 \%$ & $2.63[0.26,26.70]$ & 2009 & & & \\
\hline Peled 2010 & 13 & 57 & 16 & 65 & $13.6 \%$ & $0.93[0.49,1.76]$ & 2010 & & & . \\
\hline Radovanovic 2010 & 2 & 42 & 4 & 172 & $1.4 \%$ & $2.05[0.39,10.81]$ & 2010 & & & \\
\hline Zweifel-Schlatter 2010 & 17 & 49 & 15 & 58 & $12.5 \%$ & $1.34[0.75,2.40]$ & 2010 & & & \\
\hline Azzawi 2010 & 6 & 58 & 9 & 140 & $4.8 \%$ & $1.61[0.60,4.32]$ & 2010 & & & \\
\hline Donker 2012 & 4 & 48 & 29 & 215 & $9.6 \%$ & $0.62[0.23,1.68]$ & 2012 & & & - \\
\hline Lardi 2013 & 7 & 21 & 19 & 128 & $4.9 \%$ & $2.25[1.08,4.68]$ & 2013 & & & \\
\hline Schaverien 2013 & 11 & 33 & 19 & 62 & $12.0 \%$ & $1.09[0.59,2.00]$ & 2013 & & & $=$ \\
\hline Narui 2015 & 6 & 40 & 22 & 165 & $7.8 \%$ & $1.13[0.49,2.59]$ & 2015 & & & \\
\hline D'Alessandro 2016 & 6 & 33 & 8 & 69 & $4.7 \%$ & $1.57[0.59,4.15]$ & 2016 & & & \\
\hline Terao 2017 & 3 & 20 & 0 & 18 & $0.5 \%$ & $6.33[0.35,114.81]$ & 2017 & & & \\
\hline Beugels 2019 & 8 & 67 & 59 & 365 & $16.7 \%$ & $0.74[0.37,1.47]$ & 2019 & & & - \\
\hline Allué Cabañuz 2019 & 3 & 62 & 6 & 109 & $4.0 \%$ & $0.88[0.23,3.39]$ & 2019 & & & \\
\hline Moon 2020 & 5 & 17 & 47 & 197 & $6.8 \%$ & $1.23[0.57,2.68]$ & 2020 & & & \\
\hline Total $(95 \% \mathrm{Cl})$ & & 559 & & 1826 & $100.0 \%$ & $1.15[0.92,1.44]$ & & & & \\
\hline Total events & 92 & & 255 & & & & & & & \\
\hline $\begin{array}{l}\text { Heterogeneity: } \mathrm{Chi}^{2}=1 \\
\text { Test for overall effect: }\end{array}$ & $\begin{array}{l}30, \mathrm{df}= \\
1.21(\mathrm{P}\end{array}$ & $\begin{array}{l}13(P \\
=0.2\end{array}$ & $\begin{array}{l}=0.67) \text {; } \\
\text { 2) }\end{array}$ & $1^{2}=0 \%$ & & & & 0.0 & $\begin{array}{c}0.1 \\
\text { Favours [NACT] }\end{array}$ & 10 \\
\hline
\end{tabular}

\section{B. Wound complications* in all reconstructions}

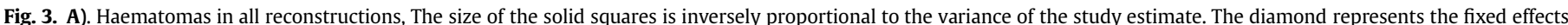

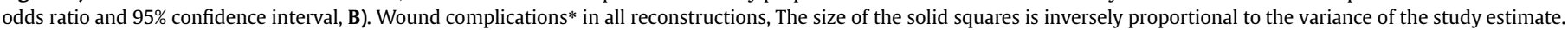

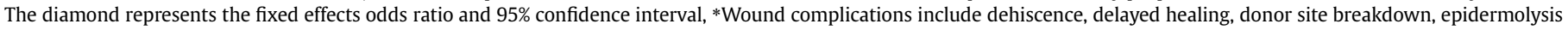
and superficial wound infection.

\begin{tabular}{|c|c|c|c|c|c|c|c|c|c|}
\hline Study or Subgroup & \multicolumn{2}{|c|}{ NACT } & \multicolumn{2}{|c|}{ Control } & Weight & $\begin{array}{c}\text { Risk Ratio } \\
\text { M-H, Fixed, 95\% Cl }\end{array}$ & \multicolumn{3}{|c|}{$\begin{array}{c}\text { Risk Ratio } \\
\text { M-H, Fixed, 95\% Cl }\end{array}$} \\
\hline Azzawi 2010 & 4 & 39 & 3 & 28 & $48.3 \%$ & $0.96[0.23,3.94]$ & & & \\
\hline Donker 2012 & 0 & 37 & 3 & 176 & $17.1 \%$ & $0.67[0.04,12.62]$ & & & \\
\hline Schaverien 2013 & 3 & 27 & 3 & 55 & $27.3 \%$ & $2.04[0.44,9.43]$ & & - & \\
\hline Terao 2017 & 3 & 20 & 0 & 18 & $7.3 \%$ & $6.33[0.35,114.81]$ & & & \\
\hline Total $(95 \% \mathrm{CI})$ & & 123 & & 277 & $100.0 \%$ & $1.59[0.66,3.87]$ & & & \\
\hline Total events & 10 & & 9 & & & & & & \\
\hline $\begin{array}{l}\text { Heterogeneity: } \mathrm{Chi}^{2} \\
\text { Test for overall effec }\end{array}$ & $\begin{array}{l}1.81, \mathrm{df} \\
\mathrm{Z}=1.0\end{array}$ & $\begin{array}{l}3(P \\
(P=\end{array}$ & $\begin{array}{l}=0.61) \\
0.30)\end{array}$ & ${ }^{2}=0 \%$ & & & $\begin{array}{cc}0.01 & 0.1 \\
\text { Favours [NACT] }\end{array}$ & $\begin{array}{c}10 \\
\text { Favours [control] }\end{array}$ & 100 \\
\hline
\end{tabular}

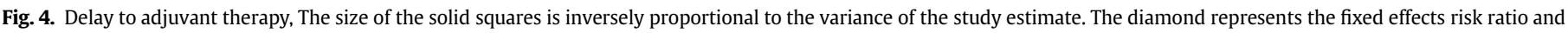
95\% confidence interval.

\section{Publication bias}

The funnel plots of overall complications are presented in Fig. 5. Visual inspection suggests that smaller studies were more likely to report a protective effect of NACT, but all points were within the funnel. Funnel plots for each meta-analysis are provided in the supplementary material and as a whole they show little evidence of publication bias.

\section{Discussion}

All studies included in this review are observational studies, as no randomized controlled trials have been performed on this subject. This meta-analysis includes the largest number of highquality studies all using similar NACT agents and regimens with the same timeline from end of NACT to surgery in line with current guidelines. While disease-free survival and overall survival are reported to be similar for women receiving neoadjuvant compared 


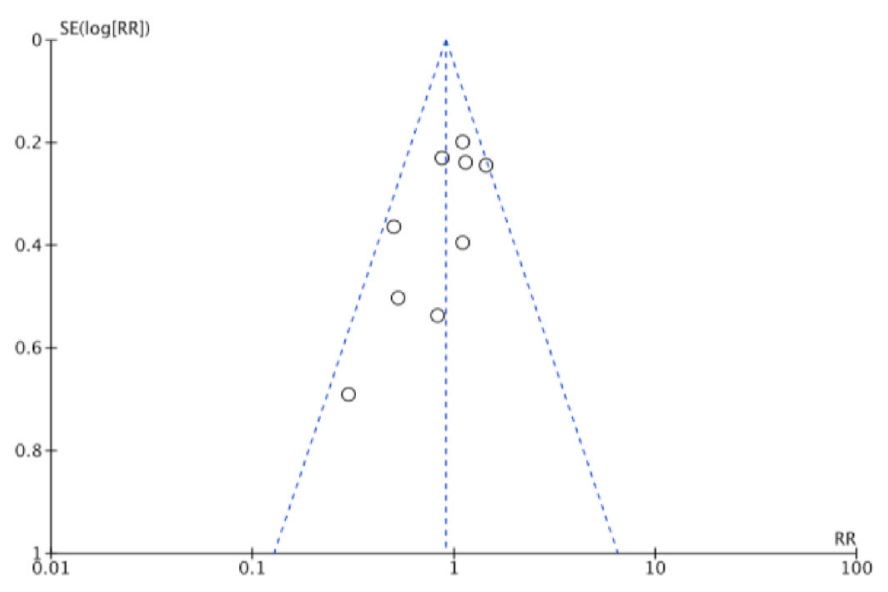

Fig. 5. Funnel plot demonstrating the absence of publication bias among the studies that reported overall complications.

with adjuvant chemotherapy $[3,35]$ a delay in the receipt of adjuvant therapy, often due to post-operative complications, has been shown to have a negative impact on patients' oncological outcome [36,37].

Chemotherapy targets proliferating cells by interfering with specific processes of the cell cycle, eliciting an effect on the immune system that can impair wound healing and increase susceptibility to infection. Multiple experimental studies performed in animals have corroborated these effects, showing decreased wound strength after the administration of chemotherapeutic agents $[12,13]$. Agents such as anthracyclines have well-documented effects on endothelial cells, potentially leading to increased risk of thrombosis [11]. Interestingly, these effects are not demonstrated in clinical practice, especially if surgery is performed at least three weeks after chemotherapy to allow for hematological parameters, including the white cell count, to recover [14]. Published data on NACT in esophageal malignancies, colorectal cancers and sarcomas has not shown higher rates of post-operative complications or anastomotic leaks [38-40]. In terms of breast cancer, Song et al. published a meta-analysis in 2014 showing better surgical outcomes among women who received NACT. The authors of that work commented upon a clearly evident selection bias in the studies, with the NACT groups containing younger and fitter patients. This systematic-review and meta-analysis builds on Songs work with better matched cohorts for comparison and utilizes newer studies assessing current chemotherapy regimens.

This study's pooled estimates indicate that rates of overall surgical complications after immediate breast reconstructions was not associated with use of NACT prior to surgery, with no significant differences in major or minor complications found between patients who did and did not receive NACT prior to their IBR. The studies included in this work ensured appropriate matching of patients in both comparative arms thereby by allowing this metaanalysis to draw generalizable and more meaningful comparisons.

This is the first meta-analysis to address the effect of NACT on surgical outcomes divided by reconstruction type. There are two main types of immediate breast reconstruction techniques: I/E and autologous (pedicle or free flaps). Complication rates are inherently different for each type of reconstruction and hence subset analyses for each type of reconstruction were done in addition to the overall complication estimation.

The effect of chemotherapeutic agents on tissue quality and structure can present surgeons with challenging perforator dissection in autologous reconstructions, as well as making fascial planes indistinct, increasing the risk of mastectomy skin necrosis
[21]. Additional pressure on skin flaps from a large volume autologous flap could theoretically increase the incidence of postoperative complications in these women. This fear of increased complications among surgeons may partially explain the reduced odds of women treated with NACT undergoing immediate breast reconstruction, even after adjusting for age, disease stage and need for adjuvant therapy [15]. Reassuringly, meta-analysis performed on data from five studies indicates that there is no statistically significant difference in flap loss rate after an autologous reconstruction among patients receiving NACT compared to those who did not receive NACT [18-22]. This is in agreement with most published studies where a direct deleterious effect of NACT on anastomotic patency is not seen, although some authors have commented on reduced tissue quality, vessel edema and technically challenging perforator dissections [21]. With advances in freeflap dissection techniques and atraumatic microsurgical techniques, adverse outcomes in large centers have been kept to an acceptable level.

Given that NACT has not shown a survival benefit over adjuvant chemotherapy, immediate breast reconstruction in these women must be performed in a manner that ensures timely access to adjuvant therapy [35,41]. A delay to adjuvant therapy of 8-12 weeks has been shown to adversely affect disease-free and overall survival [36,37]. This is even more pertinent now during the COVID-19 pandemic, when risk of complications must be minimized.

Hematomas, seromas, and wound complications are all factors which may not directly cause failure of reconstruction but may delay administration of adjuvant therapy. Nine of the 14 studies evaluated in this meta-analysis showed non-significant trends towards increased minor complications among women who had received NACT [19,21,22,24,28-30,32,33]. This is supported by the findings of Mehrara et al., who looked at 952 women of whom 70 had NACT prior to immediate reconstructions [42]. NACT was an independent predictor of post-operative complications (OR 2.1; $p<0.01$ ) and was associated with wound healing problems (OR 2.9; $p=0.02$ ) and fat necrosis (OR 2.8; $p<0.01$ ). Data from this study could not be included in this meta-analysis as the authors no longer had access to the raw data. Overall, our meta-analysis did not find a significant increase in the rate of minor complications following an IBR post-NACT compared with the rate of minor compilations when in patients who underwent an IBR not preceded by NACT. In addition, our pooled estimate did not show a significant delay in start of adjuvant therapy (RR: $1.65,95 \%$ CI 0.64 to $4.28, p=0.30$ ).

Of note, Beugels et al. reported reduced complication rates among women who had NACT before surgery [18]. The protective association in Beugels's work was thought to be at least in part related to patient selection, with significantly younger women in the NACT group and more active smokers in the control group, possibly allowing the NACT group to keep their complication level low. This meta-analysis goes some way to control for the selection bias seen in the NACT group, therefore allowing our results to be more generalizable.

The effect of age and other patient factors on breast reconstruction outcomes has been studied and shown to be particularly pertinent in implant-based reconstruction [43]. An analysis of 118 patients who underwent immediate breast reconstruction after mastectomies found that patient age was related to reconstruction failure ( $O R=3.02, p=0.02$ ) [26]. This finding may reflect a relative impairment of the wound-healing capacity in older populations [43]. Likewise, high body-mass index (BMI) is another independent risk factor for complications; for every five-unit increase in $\mathrm{BMI}$, the odds of developing complications is estimated to be 1.51 [44]. Furthermore, breasts larger than $600 \mathrm{~g}$ are associated with increased risk of infection and smokers have a higher risk of 
implant failure [44]:

The effect of minor complications may be more relevant in implant-based reconstructions, where delayed healing, wound dehiscence or infection could result in implant loss. Moreover, many complications, especially wound-related complications, are difficult to determine retrospectively and may be underestimated. Outcome measures such as implant loss rates are more objective and clinically relevant. Our pooled estimate of data from five studies showed that some evidence to suggest implant losses were more likely to occur in patients receiving NACT compared to control groups (RR: $1.54,95 \% \mathrm{CI} 1.04$ to $2.29, p=0.03$ ) [24-27,33]. It is difficult to ascertain the impact of adjuvant therapy on this outcome, as it is not always documented clearly in the papers. When data was restricted to the two studies that looked at NACT and implant loss for first 6 weeks alone, thereby removing the effect of adjuvant therapy, no significant implant loss was seen (RR: $0.92,95 \% \mathrm{CI} 0.39$ to $2.15, p=0.84)$ [25,27]. A relevant question to inform clinical practice is whether reconstructive outcomes are different for women undergoing adjuvant or neoadjuvant chemotherapy. Peled et al. reported no difference in implant losses for both these groups when compared to women that did not have chemotherapy [33]. Interestingly, in this study, fifty-nine percent of patients undergoing neoadjuvant chemotherapy had postoperative radiation therapy, compared with thirty-six percent of patients treated with adjuvant chemotherapy $(p=0.05)$. Despite this difference, no increase in implant loss was seen in women that received NACT. While this suggests that NACT does not increase risk of implant losses, larger prospective studies with accurate data collection will help inform future practice.

An inherent limitation of our study design is that when data on events are extracted from study reports, they are not adjusted for patient selection biases, as data on patient and tumour characteristics are not available. Individual-level data was not available from authors and adjusted estimates were not provided in the reports. Attributing cause and effect is also challenging as a multitude of patient and biological factors influence post-operative outcomes after an IBR. These include, but are not limited to, the incision used, patients smoking status, BMI and mastectomy weight. Raw data was not available to ensure adequate matching or comparison between the cohort of patients who received NACT prior to their IBR and those who did not. However, most studies commented upon aiming to match both cohorts and commented on any bias, most notable in the Donker et al. study as discussed above [25]. Estimating the impact of adjuvant therapy on outcomes is also not clear especially when the follow-up periods varied.

With regards to women who are planning to undergo mastectomy and immediate reconstruction, the results of our metaanalysis indicate that NACT remains a safe option that does not appear to cause significant wound complications, reconstruction loss, or subsequent delay to adjuvant therapy. This study highlights the need to consider other risk factors known to impact reconstructive outcomes in addition to NACT [45]. There may be an opportunity to utilize the time during NACT administration to optimize some of the modifiable factors such as smoking and BMI. This is especially pertinent for implant-based reconstructions. Through careful patient selection, we prioritize cancer treatment and patient safety, but keep reconstruction as a viable option for appropriate candidates, maintaining patient autonomy and aesthetics.

\section{Contributors}

JV, SG AND HR wrote the first draft. JV, SG, and MF did data extraction, data analysis, and data interpretation. BL, LJ, PS, and SK proofread and edited the manuscript.

\section{Declaration of competing interest}

The authors declare no conflict of interest.

\section{Acknowledgements}

We would like to thank Dr. Adam Brentnall, Centre for Cancer Prevention, Queen Mary University of London, for overseeing the statistical work and his helpful comments on the manuscript.

\section{Appendix A. Supplementary data}

Supplementary data to this article can be found online at https://doi.org/10.1016/j.breast.2020.11.023.

\section{Category}

Systematic review and meta-analysis.

\section{Funding source}

None.

\section{Ethical approval}

Ethical approval was not required for this study.

\section{References}

[1] Mieog JSD, Van Der Hage JA, Van De Velde CJH. Neoadjuvant chemotherapy for operable breast cancer. Br J Surg 2007. https://doi.org/10.1002/bjs.5894.

[2] Smith IE, Lipton L. Preoperative/neoadjuvant medical therapy for early breast cancer. Lancet Oncol 2001. https://doi.org/10.1016/S1470-2045(01)00490-9.

[3] Asselain B, Barlow W, Bartlett J, et al. Long-term outcomes for neoadjuvant versus adjuvant chemotherapy in early breast cancer: meta-analysis of individual patient data from ten randomised trials. Lancet Oncol 2018. https:// doi.org/10.1016/S1470-2045(17)30777-5.

[4] Liu SV, Melstrom L, Yao K, Russell CA, Sener SF. Neoadjuvant therapy for breast cancer. J Surg Oncol 2010. https://doi.org/10.1002/jso.21446.

[5] Van de Wiel M, Dockx Y, Van den Wyngaert T, Stroobants S, Tjalma WAA, Huizing MT. Neoadjuvant systemic therapy in breast cancer: challenges and uncertainties. Eur J Obstet Gynecol Reprod Biol 2017. https://doi.org/10.1016/ j.ejogrb.2016.12.014.

[6] Alberro J, Ballester B, Deulofeu P, et al. Long-term outcomes for neoadjuvant versus adjuvant chemotherapy in early breast cancer, vol. 39; 2018.

[7] Zhong T, Hu J, Bagher S, Vo A, O'Neill AC, Butler K, Novak CB, Hofer SOP, Metcalfe KA. A comparison of psychological response, body image, sexuality, and quality of life between immediate and delayed autologous tissue breast reconstruction: a prospective long-term outcome study. Plast Reconstr Surg 2016. https://doi.org/10.1097/PRS.0000000000002536.

[8] Al-Ghazal SK, Sully L, Fallowfield L, Blamey RW. The psychological impact of immediate rather than delayed breast reconstruction. Eur J Surg Oncol 2000. https://doi.org/10.1053/ejso.1999.0733.

[9] Jagsi R, Jiang J, Momoh AO, Alderman A, Giordano SH, Buchholz TA, Kronowitz SJ, Smith BD. Trends and variation in use of breast reconstruction in patients with breast cancer undergoing mastectomy in the United States. J Clin Oncol 2014. https://doi.org/10.1200/JCO.2013.52.2284.

[10] Hu ES, Pusic AL, Waljee JF, Kuhn L, Hawley ST, Wilkins E, Alderman AK. Patient-reported aesthetic satisfaction with breast reconstruction during the long-term survivorship period. Plast Reconstr Surg 2009. https://doi.org/ 10.1097/PRS.0b013e3181ab10b2.

[11] Sandoo A, Kitas GD, Carmichael AR. Endothelial dysfunction as a determinant of trastuzumabmediated cardiotoxicity in patients with breast cancer. Anticancer Res 2014

[12] Lawrence WT, Talbot TL, Norton JA. Preoperative or postoperative doxorubicin hydrochloride (Adriamycin): which is better for wound healing? Surgery 1986.

[13] Devereux DF, Thibault L, Boretos J, Brennan MF. The quantitative and qualitative impairment of wound healing by adriamycin. Cancer. 1979. https:// doi.org/10.1002/1097-0142(197903)43:3<932::AIDCNCR2820430322>3.0.CO;2-A.

[14] Kuerer HM, Hunt KK, Newman LA, Ross MI, Ames FC, Singletary SE. Neoadjuvant chemotherapy in women with invasive breast carcinoma: conceptual basis and fundamental surgical issues. J Am Coll Surg 2000. https:// doi.org/10.1016/S1072-7515(99)00272-0.

[15] Hu YY, Weeks CM, In H, et al. Impact of neoadjuvant chemotherapy on breast 
reconstruction. Cancer. 2011. https://doi.org/10.1002/cncr.25872.

[16] Moher D, Liberati A, Tetzlaff J, et al. Preferred reporting items for systematic reviews and meta-analyses: the PRISMA statement. PLoS Med 2009. https:// doi.org/10.1371/journal.pmed.1000097.

[17] Higgins JPT, Thompson SG. Quantifying heterogeneity in a meta-analysis. Stat Med 2002. https://doi.org/10.1002/sim.1186.

[18] Beugels J, Meijvogel JLW, Tuinder SMH, Tjan-Heijnen VCG, Heuts EM, Piatkowski A, van der Hulst RRWJ. The influence of neoadjuvant chemotherapy on complications of immediate DIEP flap breast reconstructions. Breast Canc Res Treat 2019. https://doi.org/10.1007/s10549-019-05241-9.

[19] Moon KC, Baek SO, Yoon ES, Lee B Il, Park SH. Predictors affecting complications and aesthetic outcomes in autologous breast reconstruction with free muscle-sparing transverse rectus abdominis myocutaneous flaps. Microsurgery 2020. https://doi.org/10.1002/micr.30442.

[20] Narui K, Ishikawa T, Satake T, et al. Outcomes of immediate perforator flap reconstruction after skin-sparing mastectomy following neoadjuvant chemotherapy. Eur J Surg Oncol 2015. https://doi.org/10.1016/ j.ejso.2014.09.001.

[21] Schaverien MV, Munnoch DA. Effect of neoadjuvant chemotherapy on outcomes of immediate free autologous breast reconstruction. Eur J Surg Oncol 2013. https://doi.org/10.1016/j.ejso.2013.02.015.

[22] Z-S M, D N, R P, R D, Z M, F J. Immediate microvascular breast reconstruction after neoadjuvant chemotherapy: complication rates and effect on start of adjuvant treatment. Ann Surg Oncol 2010.

[23] Allué Cabañuz M, Arribas del Amo MD, Gil Romea I, Val-Carreres Rivera MP, Sousa Domínguez R, Güemes Sánchez AT. Direct-to-implant breast reconstruction after neoadjuvant chemotherapy: a safe option? Cir Esp 2019. https://doi.org/10.1016/j.ciresp.2019.07.003.

[24] Lardi AM, Ho-Asjoe M, Mohanna PN, Farhadi J. Immediate breast reconstruction with acellular dermal matrix: factors affecting outcome. J Plast Reconstr Aesthetic Surg 2014. https://doi.org/10.1016/j.bjps.2014.05.020.

[25] Donker M, Hage JJ, Woerdeman LAE, Rutgers EJT, Sonke GS, Vrancken Peeters MJTFD. Surgical complications of skin sparing mastectomy and immediate prosthetic reconstruction after neoadjuvant chemotherapy for invasive breast cancer. Eur J Surg Oncol 2012. https://doi.org/10.1016/ j.ejso.2011.09.005.

[26] Jiménez-Puente A, Prieto-Lara E, Rueda-Domínguez A, Maañón-Di Leo C Benítez-Parejo N, Rivas-Ruiz F, Medina-Cano FJ, Perea-Milla E. Complications in immediate breast reconstruction after mastectomy. Int J Technol Assess Health Care 2011. https://doi.org/10.1017/S026646231100047X.

[27] Radovanovic Z, Radovanovic D, Golubovic A, Ivkovic-Kapicl T, Bokorov B, Mandic A. Early complications after nipple-sparing mastectomy and immediate breast reconstruction with silicone prosthesis: results of 214 procedures. Scand J Surg 2010. https://doi.org/10.1177/145749691009900302.

[28] Liu Y, Mori H, Hata Y. Does neoadjuvant chemotherapy for breast cancer increase complications during immediate breast reconstruction? J Med Dent Sci 2009. https://doi.org/10.11480/jmds.560106.

[29] Terao Y, Taniguchi K, Fujii M, Moriyama S. Postmastectomy radiation therapy and breast reconstruction with autologous tissue. Breast Cancer 2017. https:// doi.org/10.1007/s12282-017-0760-5.

[30] D GS, P A, dos S LKIL, M AM, G R, de Sg JC. Effect of neoadjuvant chemotherapy on women undergoing breast cancer surgery and immediate breast reconstruction with latissimus dorsi flap and silicone implants. Eur J Plast Surg 2017. https://doi.org/10.1007/s00238-016-1263-x.
[31] Lee TJ, Oh TS, Kim EK, Suh H, Ahn SH, Son BH, Lee JW, Cho J, Eom JS. Risk factors of mastectomy skin flap necrosis in immediate breast reconstruction using low abdominal flaps. J Plast Surg Hand Surg 2016. https://doi.org/ 10.3109/2000656X.2016.1170026.

[32] Azzawi K, Ismail A, Earl H, Forouhi P, Malata CM. Influence of neoadjuvant chemotherapy on outcomes of immediate breast reconstruction. Plast Reconstr Surg 2010. https://doi.org/10.1097/PRS.0b013e3181da8699.

[33] Peled AW, Itakura K, Foster RD, Hamolsky D, Tanaka J, Ewing C, Alvarado M, Esserman LJ, Hwang ES. Impact of chemotherapy on postoperative complications after mastectomy and immediate breast reconstruction. Arch Surg 2010. https://doi.org/10.1001/archsurg.2010.163.

[34] Godfrey PM, Godfrey NV, Romita MC. Immediate autogenous breast reconstruction in clinically advanced disease. Plast Reconstr Surg 1995. https:// doi.org/10.1097/00006534-199505000-00013.

[35] Mauri D, Pavlidis N, Ioannidis JPA. Neoadjuvant versus adjuvant systemic treatment in breast cancer: a meta-analysis. J Natl Cancer Inst 2005. https:// doi.org/10.1093/jnci/dji021.

[36] Gold HT, Do HT, Dick AW. Correlates and effect of suboptimal radiotherapy in women with ductal carcinoma in situ or early invasive breast cancer. Cancer. 2008. https://doi.org/10.1002/cncr.23923.

[37] Lohrisch C, Paltiel C, Gelmon K, Speers C, Taylor S, Barnett J, Olivotto IA. Impact on survival of time from definitive surgery to initiation of adjuvant chemotherapy for early-stage breast cancer. J Clin Oncol 2006. https://doi.org/ 10.1200/JCO.2005.01.6089.

[38] Minamide J, Aoyama N, Koizumi H, Yoneyama K, Hoshino S, Kamiya J, Tamai S, Kameda Y. Postoperative complications in patients of esophageal cancer after neoadjuvant chemotherapy. Jpn J Thorac Cardiovasc Surg 1999. https:// doi.org/10.1007/BF03218059.

[39] Tabira Y, Okuma T, Kondo K, Yoshioka M, Mori T, Tanaka M, Nakano K, Kitamura N. Does neoadjuvant chemotherapy for carcinoma in the thoracic esophagus increase postoperative morbidity? Jpn J Thorac Cardiovasc Surg 1999. https://doi.org/10.1007/BF03218027.

[40] Turner II, Russell GB, Blackstock AW, Levine EA. Impact of neoadjuvant therapy on postoperative complications in patients undergoing resection for rectal adenocarcinoma. Am Surg 2004.

[41] Rastogi P, Anderson SJ, Bear HD, et al. Preoperative chemotherapy: updates of national surgical adjuvant breast and bowel project protocols B-18 and B-27. J Clin Oncol 2008. https://doi.org/10.1200/JCO.2007.15.0235.

[42] Mehrara BJ, Santoro TD, Arcilla E, Watson JP, Shaw WW, Da Lio AL. Complications after microvascular breast reconstruction: experience with 1195 flaps. Plast Reconstr Surg 2006. https://doi.org/10.1097/ 01.prs.0000236898.87398.d6.

[43] Lipa JE, Youssef AA, Kuerer HM, Robb GL, Chang DW. Breast reconstruction in older women: advantages of autogenous tissue. Plast Reconstr Surg 2003. https://doi.org/10.1097/01.PRS.0000046614.84464.84.

[44] Martin L, O’Donoghue JM, Horgan K, Thrush S, Johnson R, Gandhi A. Acellular dermal matrix (ADM) assisted breast reconstruction procedures: joint guidelines from the association of breast surgery and the British association of plastic, reconstructive and aesthetic surgeons. Eur J Surg Oncol 2013. https:// doi.org/10.1016/j.ejso.2012.12.012.

[45] Woerdeman LAE, Hage JJ, Hofland MMI, Rutgers EJT. A prospective assessment of surgical risk factors in 400 cases of skin-sparing mastectomy and immediate breast reconstruction with implants to establish selection criteria. Plast Reconstr Surg 2007. https://doi.org/10.1097/01.prs.0000246379.99318.74. 